\title{
Responsabilité pour la maladie
}

\section{Samia Hurst}

Prof. Dr méd., membre de la rédaction, Institut Ethique Histoire Humanités (iEH2), Faculté de médecine, Genève

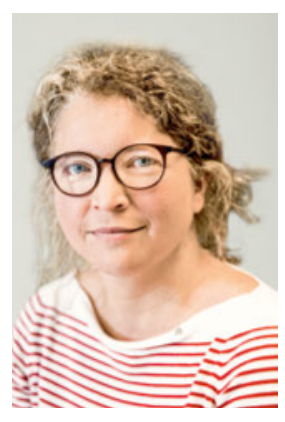

Les malades qui ne prennent pas leur traitement correctement génèrent des surcoûts, nous devrions les sanctionner. L'exemple est tiré de la presse récente. Mais en réalité, ce genre de proposition est récurrent. Infliger des malus (ou des bonus) selon que l'on adopte un comportement bon ou mauvais pour la santé est dans l'air du temps. Certains pays plus totalitaires que le nôtre, comme la Hongrie, avaient décidé de réserver les médicaments les plus efficaces aux patients les mieux disciplinés. Plus près de nous, on avait il y a quelques années discuté de réserver l'accès aux antiviraux onéreux visant l'hépatite $C$ aux seuls patients qui seraient «innocents» de leur maladie.

Si la responsabilisation est ainsi à l'ordre du jour, ce n'est pas étonnant. L'idée de la responsabilité personnelle, du mérite individuel, est un des mythes de notre culture. C'est une des fables que notre société se raconte. Un récit organisateur dont le but est de nous aider à comprendre le monde et à le justifier. Dans ce récit, notre situation professionnelle et personnelle, notre fortune, notre revenu, notre bonheur même et bien sûr notre santé dépendent principalement de notre mérite. Dans ce récit, nos efforts sont les piliers principaux de tous nos succès, et nos échecs signalent de manière infaillible autant d'occasions où nous n'avons pas donné le meilleur de nous-mêmes. Ce discours est très présent quand on touche à notre santé. Une des raisons est qu'il comporte une part de vérité. A mesure que les maladies les plus prévalentes sont liées au mode de vie, nous pouvons effectivement faire quelque chose pour notre santé. C'est une bonne nouvelle. Les messages de santé publique peuvent être utiles.

L'ennui, c'est que le récit selon lequel nous serions dès lors pleinement responsables de notre santé est, lui, une fable. Notre capacité à vivre de manière saine est fortement influencée par toute une foule de paramètres qui, eux, échappent à notre contrôle. Si vous avez grandi à une période où la publicité pour le tabac auprès des jeunes était moins bien réglementée, vous aurez peutêtre du mal à redevenir aujourd'hui libre d'un produit addictif. Si votre journée de travail est longue, vous serez peut-être trop sédentaire à votre place de travail. Si vous avez du mal à boucler vos fins de mois, votre quote-part pour ce médicament vous poussera peutêtre à remettre son achat au mois prochain. Selon le temps et l'argent à disposition, la qualité de votre alimentation s'en ressentira peut-être aussi. Le degré de contrôle sur notre propre vie est en fait directement associé à la santé, et il n'est pas réparti de manière égale.

\section{Notre capacité à vivre de manière saine est} fortement influencée par toute une foule de paramètres qui, eux, échappent à notre contrôle.

La responsabilité pour la santé n'est pas inexistante, donc. On peut tabler sur elle. En revanche, comment, dans ces conditions, vouloir sanctionner les «mauvais malades»? En établissant la part de responsabilité personnelle et en reconnaissant des circonstances atténuantes? Devant quel tribunal et selon quelles règles? Y aura-t-on droit à la présomption d'innocence, à un avocat (ou un médecin peut-être) pour assurer notre défense? Lorsque le dossier médical devient un casier judiciaire, on a tendance à oublier que la justice vient, elle aussi, avec ses règles de l'art. 